\title{
Criminologie
}

\section{Les enjeux à venir pour la criminologie clinique : approche développementale et intégration avec les sciences biomédicales}

\section{René Carbonneau}

Volume 41, numéro 1, printemps 2008

40 ans de Criminologie : perspectives d'avenir

URI : https://id.erudit.org/iderudit/018419ar

DOI : https://doi.org/10.7202/018419ar

Aller au sommaire du numéro

\section{Éditeur(s)}

Les Presses de l'Université de Montréal

ISSN

0316-0041 (imprimé)

1492-1367 (numérique)

Découvrir la revue

Citer cet article

Carbonneau, R. (2008). Les enjeux à venir pour la criminologie clinique : approche développementale et intégration avec les sciences biomédicales. Criminologie, 41(1), 47-82. https://doi.org/10.7202/018419ar

\section{Résumé de l'article}

$\mathrm{Au}$ cours des dernières décennies, la criminologie clinique a connu un essor qui annonce des changements importants pour la recherche et l'intervention à venir. Les études longitudinales sur les facteurs de risque, sur l'apparition des comportements antisociaux et sur leur trajectoire à travers la vie des individus ont d'abord produit un ensemble de connaissances qui ont permis une meilleure compréhension du phénomène de la délinquance dans une perspective développementale. Puis, l'évolution fulgurante de la recherche en sciences biomédicales et son impact sur la compréhension de l'étiologie et sur le traitement des problèmes de santé mentale, lesquels recoupent de façon importante les comportements antisociaux, ont entraîné l'émergence du paradigme bio-psycho-social comme base d'étude et d'intervention en criminologie clinique. Les résultats des études en neurosciences et en génétique du comportement sont particulièrement éloquents quant à l'utilité de ce paradigme pour aborder dans sa totalité le phénomène délinquant. $\mathrm{Si}$ l'avènement de l'approche bio-psycho-sociale dans un cadre développemental et son impact sur la compréhension du comportement antisocial sont récents, les possibilités sans précédent qui s'offrent maintenant aux chercheurs et aux intervenants en criminologie clinique rendent la poursuite active de cette nouvelle stratégie incontournable.
Ce document est protégé par la loi sur le droit d'auteur. L'utilisation des services d'Érudit (y compris la reproduction) est assujettie à sa politique d'utilisation que vous pouvez consulter en ligne.

https://apropos.erudit.org/fr/usagers/politique-dutilisation/ 


\title{
Les enjeux à venir pour la criminologie clinique : approche développementale et intégration avec les sciences biomédicales
}

\author{
René Carbonneau \\ Département de Pédiatrie, Faculté de Médecine, Université de Montréal \\ Centre de recherche du CHU Sainte-Justine \\ Groupe de Recherche sur l'Inadaptation Psychosociale chez l'enfant (GRIP) \\ Centre d'Excellence en Neuromique (CEN), Université de Montréal \\ Réseau de Médecine Génétique Appliquée du FRSQ (RMGA) \\ rene.carbonneau@umontreal.ca
}

RÉSUMÉ - Au cours des dernières décennies, la criminologie clinique a connu un essor qui annonce des changements importants pour la recherche et l'intervention à venir. Les études longitudinales sur les facteurs de risque, sur l'apparition des comportements antisociaux et sur leur trajectoire à travers la vie des individus ont d'abord produit un ensemble de connaissances qui ont permis une meilleure compréhension du phénomène de la délinquance dans une perspective développementale. Puis, l'évolution fulgurante de la recherche en sciences biomédicales et son impact sur la compréhension de l'étiologie et sur le traitement des problèmes de santé mentale, lesquels recoupent de façon importante les comportements antisociaux, ont entraîné l'émergence du paradigme bio-psycho-social comme base d'étude et d'intervention en criminologie clinique. Les résultats des études en neurosciences et en génétique du comportement sont particulièrement éloquents quant à l'utilité de ce paradigme pour aborder dans sa totalité le phénomène délinquant. Si l'avènement de l'approche bio-psycho-sociale dans un cadre développemental et son impact sur la compréhension du comportement antisocial sont récents, les possibilités sans précédent qui s'offrent maintenant aux chercheurs et aux intervenants en criminologie clinique rendent la poursuite active de cette nouvelle stratégie incontournable.

ABSTRACT - In the last decades, clinical criminology has undergone a significant evolution which announces important changes to come for research and intervention 
in the field. Longitudinal studies on risk factors, age at onset and trajectories of antisocial behaviour throughout development have resulted in an important body of knowledge allowing for a better understanding of delinquent behaviour in a developmental perspective. Furthermore, the striking evolution of research in biomedical sciences and its impact on our understanding of the etiology and on the treatment of mental health problems, notoriously associated with criminal behaviour, have seen the bio-psycho-social paradigm emerging as the basis of research and intervention for clinical criminologists. Results of studies in neurosciences and behaviour genetics especially underscore the importance of this paradigm to fully understand delinquency. If the emergence of the bio-psycho-social paradigm in a developmental perspective and its impact on our understanding of antisocial behaviour are relatively recent, the tremendous possibilities it opens now to researchers and clinicians make this new strategy essential in the field of clinical criminology.

\section{Introduction}

La criminologie clinique a connu des changements importants au cours de la fin $\mathrm{du} \mathrm{xx}^{\mathrm{e}}$ siècle. Ces changements touchent principalement la compréhension des facteurs expliquant le comportement criminel et, conséquemment, la façon de traiter les délinquants. D’abord, l'approche développementale centrée sur le criminel s'est étendue pour couvrir l'ensemble du développement de l'individu, de la naissance, voire de la grossesse, à l'âge adulte. Puis, les progrès réalisés dans les sciences biomédicales, en particulier les neurosciences et la génétique, ont fourni des pistes explicatives inédites pour comprendre le comportement déviant. Ces pistes s'inscrivent dans un paradigme bio-psycho-social et développemental et soutiennent le nécessaire recours à une telle perspective pour comprendre, prévenir et traiter les délinquants. La criminologie $d u \mathrm{xxI}^{\mathrm{e}}$ siècle n'en est qu'à ses premiers pas que déjà on voit émerger ces champs de recherche comme pouvant révolutionner l'intervention clinique. Les sections qui suivent font le point sur l'évolution récente des connaissances dans ces domaines et soulèvent les défis et les enjeux potentiels pour l'avenir de la recherche et des pratiques en criminologie.

L'approche développementale de la délinquance:

de l'adolescence... à la naissance!

Depuis les résultats des études pionnières menées durant la première moitié du xx siècle (Glueck et Glueck, 1950; McCord et al., 1959), la recherche criminologique a permis d'identifier une variété de facteurs 
criminogènes (par exemple âge, sexe, famille, alcoolisme parental, fréquentations de pairs déviants, consommation d'alcool ou de drogues, maladies mentales, etc.), en ayant recours à une variété de stratégies méthodologiques (études corrélationnelles, transversales ou longitudinales, génogrammes, études d'adoption ou de jumeaux, etc.). Dans ce contexte de l'avancement progressif des connaissances, l'approche développementale de la délinquance, s'appuyant sur les données des études longitudinales, a joué un rôle de premier plan en permettant d'identifier les facteurs de risque et de comprendre les mécanismes influençant l'acquisition, le maintien, l'aggravation ou le désistement des comportements délinquants à travers le développement des individus. L'évolution des connaissances sur la genèse des comportements antisociaux a conduit à de nouvelles études longitudinales débutant plus tôt dans le développement et couvrant de façon plus large les différentes dimensions de l'individu. Il y a 30 ans à peine, les chercheurs soucieux d'examiner les antécédents individuels et familiaux des criminels adultes et des adolescents délinquants estimaient qu'évaluer des enfants à partir de l'âge de huit ou dix ans permettrait de se positionner bien en amont du phénomène (Huesman et al., 1984; Stattin et Magnusson, 1989; Farrington, 1994; Loeber, 1996). La raison pour laquelle on recrutait des enfants de cet âge est simple: la courbe âgecrime classique indiquait avec constance à travers nombre d'études que les comportements délinquants débutaient à partir de l'âge de dix ans et augmentaient rapidement pour atteindre un sommet vers l'âge de 16 ou 17 ans, pour ensuite décliner progressivement jusqu'au début de la vingtaine, puis au cours de l'âge adulte (Thornberry, 1997). Ainsi, le suivi longitudinal des enfants à partir du milieu ou de la fin de l'école primaire apparaissait bien suffisant pour identifier les facteurs en jeu dans le développement des comportements délinquants. Cette position développementale constituait un pas en avant important en comparaison des théories criminologiques classiques, en particulier celles d'inspiration sociologique, plus statiques pour la compréhension du délinquant et des comportements criminels, en permettant l'identification de facteurs de risque à différents moments du développement (Thornberry, 1997 ; Collins, 2004). Cependant, les résultats des études longitudinales mises sur pied au cours de cette période allaient converger vers une conclusion unanime: 1) les origines du comportement antisocial chronique étaient observables dès le premier temps d'évaluation des études, soit à six, huit ou dix ans et, en conséquence, 2) la connaissance et 
l'explication des mécanismes qui conduisent l'individu à la délinquance nécessitaient des études commençant bien plus tôt dans le développement des enfants, soit dès la naissance, voire, dès la période intra-utérine (Farrington et Coid, 2003 ; Rutter et al., 2006).

Un modèle explicatif ayant soulevé beaucoup d'intérêt dans le domaine criminologique, proposé par Moffitt (1993), s'appuie sur la définition de deux sous-types d'individus sur le plan du développement des comportements antisociaux: ceux qui présentent ces comportements tôt (early onset), soit avant l'âge de dix ans, et ceux dont les comportements apparaissent plus tard et qui sont généralement restreints à l'adolescence (late onset). Selon ce modèle, les enfants qui adoptent des comportements antisociaux tôt dans leur développement se distingueraient par une histoire de troubles du comportement, et un environnement familial et social davantage perturbé que leurs pairs délinquants à l'adolescence, pour qui la délinquance serait plutôt assimilable à une crise passagère. Les jeunes délinquants seraient aussi ceux dont la carrière délinquante serait la plus longue et les délits plus fréquents, plus réguliers et plus diversifiés. Ce modèle a depuis été soutenu par les résultats de plusieurs études longitudinales (Eley et al., 1999; Moffitt, 2003; Moffitt et Walsh, 2003). D'ailleurs, le DSM-IV (American Psychiatric Association [APA], 2000) a introduit cette distinction, sur le plan de la description et de la sévérité, entre un âge d'apparition à l'enfance ou à l'adolescence, en proposant deux sous-types pour sa catégorie diagnostique de troubles de la conduite, laquelle s'apparente le plus aux comportements délinquants.

Les études subséquentes portant sur les trajectoires des comportements antisociaux du préscolaire à l'âge adulte ont permis d'identifier un groupe d'enfants à haut risque présentant avec stabilité des comportements déviants. Ces observations ont été faites à partir d'échantillons québécois, canadiens, états-uniens et néo-zélandais (Nagin et Tremblay, 1999; Carbonneau, 2002; Broidy et al., 2003; Tremblay et al., 2004; Shaw et al., 2005 ; Carbonneau et al., 2006; Côté et al., 2006; Fontaine et al., sous presse) Ainsi, les cas chroniques de comportements antisociaux montrent des antécédents dès les premières années de vie, ce qui, pour plusieurs chercheurs, suggère une influence génétique (Lahey et al., 2003; Rutter et al., 2006; Stoff et Susman, 2005a; Tremblay et al., 2005). De plus, les études prédictives de ces trajectoires ont montré que différentes caractéristiques présentes dès la naissance ou la grossesse de la mère étaient associées au développement ultérieur des 
enfants. La consommation de tabac durant la grossesse, les comportements antisociaux antérieurs, un faible niveau d'éducation et un jeune âge pour donner naissance chez les parents ont été associés aux trajectoires chroniques de comportements antisociaux des enfants (Nagin et Tremblay, 2001; Tremblay et al., 2004; Côté et al., 2006; Romano et al., 2006).

Les résultats de ces études sur les trajectoires développementales, en soulignant la manifestation précoce des comportements antisociaux et leur stabilité à travers le temps, de même que la présence de facteurs de risque dès la grossesse, sont cohérents avec les connaissances issues d'autres études qui ont examiné les facteurs de risque en lien avec la délinquance et les troubles de comportement à différentes périodes du développement. En effet, bon nombre d'études ont clairement identifié l'influence importante des facteurs périnataux (Raine, 1993; 2005; Arseneault et al., 2002; Brennan et al., 2003) et des facteurs familiaux (Connor, 2002; Lahey et al., 2003; Rutter et al., 2006) dans le développement de ces problèmes. Il est particulièrement intéressant de noter que les mêmes facteurs périnataux et familiaux associés aux comportements délinquants adolescents (Paterson, 1982; Farrington, 2002) sont associés aux troubles du comportement au préscolaire et aux trajectoires développementales de ces comportement entre la petite enfance et l'âge adulte (Nagin et Tremblay, 2001; Tremblay et al., 2004; Côté et al., 2006; Romano et al., 2006).

Les résultats des recherches sur l'étiologie des troubles du comportement, dont le trouble de la conduite et l'hyperactivité, convergent également avec ceux des études portant plus directement sur la délinquance. Ces résultats soutiennent aussi la distinction proposée par le modèle de Moffitt (1993) sur les comportements antisociaux, et par le DSM (APA, 2000) pour la définition de la catégorie diagnostique du trouble de la conduite, en particulier pour le sous-type d'individus présentant des problèmes de comportement dès l'enfance. En ce sens, les études en criminologie développementale et clinique sont proches de la recherche en santé mentale. Or, l'évolution récente de la recherche dans ce domaine vient soutenir les résultats observés dans les études longitudinales et, conséquemment, l'importance d'adopter une perspective développementale pour la compréhension du comportement antisocial et pour l'intervention auprès des délinquants. Les résultats issus de ce courant d'étude, que nous allons maintenant aborder, devraient influencer significativement, pour les années à venir, le cours de la recherche et de l'intervention 
en criminologie clinique, en favorisant notamment le recours à une approche développementale bio-psycho-sociale.

\section{La recherche en criminologie clinique et en santé mentale: une évolution convergente}

Le lien important entre la délinquance et la santé mentale des individus a été établi dans plusieurs études (Hodgins, 1992; 2001; Hirschfield et al., 2006). Qu'il s'agisse de troubles de la personnalité (en particulier, de la personnalité antisociale, borderline ou narcissique), de dépendance ou d'abus d'alcool ou de différentes drogues, de dépression ou de troubles bipolaires, de trouble de l'impulsivité et du contrôle, de pyromanie, de jeu pathologique, d'abus physique ou sexuel, de paraphilie (dont la pédophilie et le sadisme sexuel) ou de schizophrénie chez les adultes ou, chez les enfants, de troubles de la conduite, de déficit de l'attention avec hyperactivité ou de trouble oppositionnel, pratiquement chaque catégorie générale de désordres du DSM-IV (APA, 2000) inclut des sous-catégories diagnostiques qui ont été clairement associées au comportement délinquant ou qui représentent en elles-mêmes un acte criminel. Une revue récente des études examinant la prévalence de troubles psychiatriques dans la population criminelle de différents pays conclut d'ailleurs que pratiquement personne n'échappe à une forme ou à une autre de diagnostic, le plus courant étant la personnalité antisociale (Andrews et Bonta, 2003). Cela serait d'autant plus vrai pour les délinquants violents et les délinquants précoces (Hodgins, 1992; Arseneault et al., 2000; Hodgins et Muller-Isbesner, 2000). Au Québec, un détenu sur deux souffrirait de maladie mentale et un sur trois aurait été traité à l'aide de médicaments pour ces problèmes (Le Protecteur du citoyen, 2006). D'ailleurs, les méthodes d'intervention en criminologie clinique incluent souvent un traitement médical basé sur une forme ou une autre de médication (Muller-Isberner et Hodgins, 2000; Connor, 2002).

Or, la tangente prise par l'évolution récente de la recherche clinique et fondamentale en santé mentale est sans équivoque. En effet, les progrès des neurosciences et de la génomique au cours des vingt dernières années ont vu la place de la psychiatrie génétique s'accroître substantiellement dans la recherche psychiatrique (Insel, 2003 ; Kendler, 2005). Il est maintenant largement admis: 1) que les troubles psychiatriques doivent être considérés comme des maladies du cerveau dont 
les bases génétiques sont probablement complexes et 2) que leur compréhension nécessite l'étude des conditions environnementales et la définition de phénotypes précis (Kandel, 1998; Rutter, 2002; 2005; Insel et Quirion, 2005). Parallèlement, les résultats des études en épidémiologie psychiatrique et en psychopathologie développementale ont convergé pour identifier un âge d'apparition tôt dans le développement des individus, soit à l'adolescence ou à l'enfance, pour les troubles psychiatriques observés chez les adultes (Costello et al., 2003; 2005 ; Fergusson et al., 2004; Kessler et al., 2006). Plus encore, l'étude récente de ces phénomènes au préscolaire indique qu'ils sont observables dès les premières années de vie (Gadow et al., 2001; Keenan et Shaw, 2003 ; Keenan et Wakchlag, 2004; Egger et Angold, 2006) et associés aux conditions de l'environnement des enfants, notamment les caractéristiques des parents et leurs pratiques éducatives (Tremblay et al., 2004; Lavigne et al., 1996; Romano et al., 2006).

La précocité des phénomènes de santé mentale observés et leur développement à travers le temps est donc cohérente avec les résultats des études en neurosciences et en génétique. Un parcours de recherche semblable, bien qu'à moins grande échelle, a été suivi par les études en criminologie clinique, alors que les résultats des études longitudinales dont nous avons parlé plus tôt ont conduit les chercheurs à s'intéresser davantage aux dimensions neuropsychologiques et génétiques comme facteurs d'influence sur le comportement délinquant.

\section{Neurosciences et comportement: l'importance des endophénotypes}

Le système neurobiologique humain est constitué du cerveau et de la moelle épinière. Le cerveau lui-même contient près de 100 milliards de neurones. Or, les connections entre ces neurones constituent la base de tout comportement. Les progrès réalisés à la fin $\mathrm{du} \mathrm{xx}^{\mathrm{e}}$ siècle dans la compréhension des mécanismes neuro-cérébraux ont fait des neurosciences une perspective incontournable pour la compréhension des problèmes de comportement et de santé mentale (Insel et Quirion, 2005). Essentiellement, on considère les phénomènes neuropsychologiques ou neuropsychiatriques sous-jacents aux troubles du comportement comme des endophénotypes, soit des mécanismes sous-tendant, sur le plan cérébral, le comportement (ou phénotype). La recherche sur les endophénotypes est considérée comme une stratégie prometteuse pour 
l'étude de la relation entre les gènes, le cerveau et le comportement (Rutter, 2006; Viding et Blakemore, 2007). Une revue des études en neurosciences pertinentes à la compréhension du comportement délinquant dépasse bien sûr largement le cadre du présent article. Cependant, quelques exemples suffisent à souligner l'importance de ce courant d'étude pour la recherche future en criminologie clinique. Les résultats d'études portant sur les neurotransmetteurs et les processus neuropsychologiques sont, à ce titre, particulièrement éloquents.

\section{Neurotransmetteurs et comportement délinquant}

Sur le plan des neurotransmetteurs, l'étude de la sérotonine a sans doute produit les résultats les plus frappants. La sérotonine, qui agit au niveau du système nerveux central, en particulier du lobe frontal et du système limbique, est associée à la prise de décisions, au jugement, à la régulation des émotions, à l'impulsivité et à l'agressivité (Davidson et al., 2000; Lesch, 2003; Quadagno, 2003). À titre d'exemple, un faible niveau de sérotonine a été observé en relation avec l'agressivité chez des enfants référés en clinique (Beitchman et al., 2006). Les enfants possédant le polymorphisme associé à une faible activité sérotoninergique étaient 2,7 fois plus susceptibles de présenter des comportements agressifs que leurs pairs. Un faible niveau de sérotonine a aussi été observé chez les délinquants juvéniles violents (Blumensohn et al., 1995) de même que chez des adultes agressifs (Coccaro, 1998; Siever et Trestman, 1993), ou ayant impulsivement commis un homicide involontaire ou allumé un incendie criminel (Berman et Coccaro, 1998; Fogel et al., 2000). Par ailleurs, un traitement au lithium visant à augmenter le niveau de sérotonine a entraîné une réduction significative du nombre d'agressions chez des prisonniers impulsifs-agressifs, bien que cet effet ait disparu une fois la prise de médication terminée (Coccaro, 1998).

Si la sérotonine favorise le contrôle du comportement, un niveau élevé de dopamine, en revanche, serait associé au comportement agressif et impulsif (Fishbein, 2001). La dopamine est liée à la satisfaction ou à la récompense associée à un comportement. Une stimulation de l'environnement peut ainsi entraîner un comportement conditionné à travers l'activation du système dopaminergique (Clark et Grunstein, 2000). Lorsque ce système est activé, la recherche de sensations, de nouveauté ou de stimulations augmente, ce qui pourrait favoriser un 
comportement antisocial ou déviant (Fishbein, 2001). Toutefois, bien que plusieurs études animales aient rapporté un lien entre un niveau élevé de dopamine et le comportement agressif, les observations avec les humains sont plus ambiguës (Raine, 1993; Coccaro et al., 2000). Par ailleurs, la dopamine est traditionnellement associée au trouble du déficit de l'attention avec hyperactivité (TDAH) (Voeller, 2006), lequel est un des prédicteurs principaux du comportement antisocial chez les enfants (Comings, 2000). Les critiques méthodologiques des études connues sur l'association entre la dopamine et le comportement délinquant (Fishbein, 2001) et le lien important entre ce neurotransmetteur et le TDAH font de ce champ d'étude une piste qui demeure à être explorée dans la recherche future en criminologie clinique.

La norépinéphrine est également un neurotransmetteur du système nerveux central, associé aux émotions, à l'attention et à l'excitation et impliqué dans la transmission de l'information au système nerveux périphérique. Une augmentation de ce neurotransmetteur est liée à une activité demandant un niveau d'éveil ou d'excitation élevé, incluant le comportement violent (Quadagno, 2003). Comme la dopamine, dont elle est le produit, la norépinéphrine a été associée au comportement agressif chez les animaux et chez les humains (Filley et al., 2001; Mattson, 2003). Par exemple, on rapporte une corrélation positive entre la norépinéphrine et l'histoire d'agression ou l'impulsivité chez des sujets présentant un trouble de la personnalité (Brown et al., 1979; Siever et Trestman, 1993).

Pour illustrer la complexité de la recherche sur la relation entre les neurotransmetteurs et le comportement délinquant et les défis importants à relever dans ce domaine, terminons en notant que la monoamine oxydase (MAOA), une enzyme responsable de la dégradation de plusieurs neurotransmetteurs, dont la sérotonine, la dopamine et la norépinéphrine, a été associée avec régularité dans la littérature avec le comportement antisocial, l'alcoolisme, la recherche de sensations et l'impulsivité (Shih et Thompson, 1999; Eklund et al., 2005). Ainsi, l'influence spécifique des différents neurotransmetteurs, leurs éventuelles interactions et le rôle d'éléments faisant partie du processus biologique de la neurotransmission, comme la MAOA, nécessiteront davantage de recherche pour en comprendre la place dans l'étiologie du comportement délinquant. L'étude des neurotransmetteurs avec une perspective développementale sera également importante. Collins (2004), suggère que la courbe âge-crime et le désistement «naturel» observé chez les 
criminels adultes peuvent s'expliquer par le fait que le niveau de sérotonine augmente avec l'âge, ce qui aurait un effet modérateur sur les comportements violents et sur l'impulsivité, alors que la dopamine et la norépinéphrine, qui favoriseraient ces comportements (Coccaro et al., 2000), diminuent.

\section{Processus neuropsychologiques}

Les processus associés à la régulation des fonctions cognitives et des émotions ont fait l'objet de nombreuses études. Parmi celles-ci, plusieurs ont examiné le comportement antisocial. Les résultats de ces études convergent à identifier les habiletés cognitives, en particulier les fonctions exécutives, comme éléments clés du processus qui amène un individu à commettre des actes antisociaux, en particulier, des actes violents (Moffitt, 1993 ; 2003 ; Giancola, 2000; Raine, 2002; Nigg et Huang-Pollock, 2003 ; Séguin et Zelazzo, 2005). Les fonctions exécutives, situées au niveau du lobe frontal, sont associées, sur le plan cognitif, aux problèmes d'attention et de concentration, aux habiletés verbales, au raisonnement abstrait, à la résolution de problèmes, de même qu'à la planification de tâches et à la poursuite des buts (Fishbein, 2003). On fait l'hypothèse que des difficultés sur le plan des fonctions exécutives peuvent nuire à l'interprétation des situations sociales et des interactions avec les autres, ce qui pourrait entraîner une perception erronée de menace ou d'hostilité et conduire au comportement agressif (Giancola, 2000). En conséquence, les fonctions exécutives sont considérées comme sous-tendant les habiletés sociales, ce qui expliquerait leur lien avec les troubles de comportement chez les enfants et les comportements délinquants juvéniles et adultes (Fishbein, 2001; Ishikawa et al., 2003). Toutefois, les mécanismes exacts impliqués dans ce lien, soit la relation entre les fonctions exécutives, la régulation des émotions, les différents neurotransmetteurs et les systèmes cérébraux en jeu et, surtout, l'impact des facteurs environnementaux dans leur développement devront faire l'objet de recherches ultérieures pour être bien compris (Giancola, 2000; Nigg et Huang-Pollock, 2003; Tremblay et al., 2005). De plus, comme pour la recherche en santé mentale (Kandel, 1998; Insel et Quirion, 2005), l'idée de définir des phénotypes - comportements ou syndromes - décrivant les comportements antisociaux avec davantage de spécificité demeure un objet d'étude important en soi (Gendreau et Archer, 2005; Vitaro et Brendgen, 
2005). L'étude récente de Barker et collaborateurs (2007) est éloquente sur ce plan et sur le lien à faire avec les fonctions exécutives. Ces chercheurs ont en effet montré que les comportements de vol et de violence, lesquels sont agrégés dans les échelles de délinquance et dans les symptômes du trouble de la conduite du DSM (APA, 2000), sont distinctement associés aux fonctions exécutives: les adolescents et jeunes adultes qui commettent des vols réussissent mieux différentes tâches que ceux qui commettent des actes de violence. Ces résultats soulignent, d'une part, l'importance des endophénotypes (ici, les fonctions exécutives) pour comprendre le comportement et, d'autre part, la nécessité de définir des phénotypes suffisamment spécifiques (par exemple, comportements de violence vs vols) pour en étudier l'étiologie.

Enfin, comme nous en parlerons dans la section suivante en rapport avec les études génétiques, les progrès technologiques des dernières années ont grandement amélioré les moyens à la disposition des chercheurs pour l'étude des processus neuropsychologiques. En particulier, la technique d'imagerie par résonance magnétique (IRM), qui permet d'avoir une vue du cerveau en deux ou trois dimensions et d'en observer les différents tissus avec des contrastes très élevés, offre la possibilité de réaliser une analyse autant structurale que fonctionnelle, lorsque le cerveau est examiné alors que l'individu est soumis à une situation particulière. Cette technique a permis d'établir des liens entre, d'une part, le comportement violent et, d'autre part, des lésions ou un plus faible volume au niveau du lobe frontal (Paus, 2005), site des fonctions exécutives. Bien que les recherches à ce jour aient davantage porté sur des individus présentant des comportements violents extrêmes, l'IRM constitue un nouveau moyen d'étude précieux pour la recherche future sur les comportements antisociaux.

En somme, par les progrès importants réalisés au cours de la fin du $\mathrm{xx}^{\mathrm{e}}$ siècle, les neurosciences constituent une piste d'avenir pour la recherche criminologique. Le lien entre les mécanismes neurobiologiques et le comportement antisocial chez les humains ne fait l'objet d'études que depuis peu et de nombreuses hypothèses restent à explorer. Les liens entre ces mécanismes et d'autres éléments du fonctionnement biologique de l'humain, les systèmes endocriniens et hormonaux, par exemple, pour lesquels des résultats significatifs ont également été observés en relation avec le comportement antisocial (Brown et al., 2005; Raine, 2005; Van Goozen, 2005) demeurent à étudier. Mais, en plus de l'intérêt que représentent les connaissances produites par les 
neurosciences, le lien direct entre les endophénotypes, mécanismes sous-jacents au comportement antisocial, et certains facteurs génétiques, ajoutent à l'intérêt de ce champ d'étude pour la recherche future.

\section{Génétique du comportement délinquant: pour en finir avec le criminel-né !}

Il est étonnant, pour un chercheur contemporain, de constater que pratiquement tous les ouvrages dédiés aux théories de la délinquance qui abordent la perspective biologique font référence d'abord aux travaux de Lombroso sur le criminel-né, qualifié par l'auteur «d'atavisme» puisque présentant des caractéristiques physiques, mentales et comportementales innées qui l'assimileraient à l'homme primitif et le rendraient inapte à la vie «civilisée» (Akers, 1994; Shoemaker, 2000; Adler et al., 2001). Pourtant, l'orientation des études génétiques des dernières décennies est tout autre. Il ne s'agit plus d'identifier le criminel en puissance à partir d'une constitution génétique spécifique. Cette idée a été abandonnée il y a longtemps et il est maintenant largement reconnu que la recherche « $\mathrm{du}$ » gène expliquant des problèmes de santé mentale ou de comportement complexes comme la délinquance constitue une stratégie de recherche inappropriée (Kendler, 2005). Cela serait particulièrement vrai en ce qui concerne le comportement antisocial (Gottesman et Goldsmith, 1994; Wasserman et Wachbroit, 2001 ; Carey et Gottesman, 2006). En fait, les gènes ne sont plus considérés comme des déterminants du développement, mais bien comme interagissant avec l'environnement, qui constitue un élément essentiel à l'expression génétique et à l'acquisition de comportements (Susman et Stoff, 2005). Ce constat est issu de la recherche en génétique du comportement, laquelle a grandement progressé au cours des dernières années.

D'une part, les études du comportement antisocial basées sur des échantillons de jumeaux se sont multipliées. Une méta-analyse récente de Rhee et Waldman (2002) montre que, dans l'ensemble, ces études situent l'héritabilité des comportements antisociaux à $40 \%$. Cependant, l'héritabilité estimée varie selon les comportements considérés et les populations étudiées. Ainsi, la délinquance violente serait davantage héritable que la délinquance générale ou la délinquance non violente (Eley et al., 1999), alors que l'héritabilité estimée à l'intérieur d'un échantillon de délinquants précoces serait plus grande que chez les délinquants qui adoptent ces comportements à l'adolescence (Moffitt et 
Walsh, 2003). Bien sûr, les études de jumeaux permettent d'estimer l'influence génétique de façon indirecte et il n'est pas question ici de marqueurs spécifiques à un gène ou à un autre. Les études en génétique moléculaire permettent ce genre d'inférence. Or, si ces études ont été traditionnellement limitées par la connaissance du génome et par les moyens technologiques pour effectuer les analyses associées au génotypage et à l'identification des marqueurs et des polymorphismes génétiques, les vingt dernières années ont vu des progrès techniques fulgurants offrir des possibilités inimaginables il y a quelques temps à peine. Aussi, les études génétiques moléculaires examinant l'effet de polymorphismes spécifiques sur le comportement humain se sont multipliées. S'appuyant sur les résultats concluants de la recherche animale, notamment sur le plan de l'agressivité (Suomi, 2003; 2005a; 2005b; Pérusse et Gendreau, 2005) ces études, bien que récentes, ont montré des résultats significatifs. Le trouble de déficit de l'attention avec hyperactivité (TDAH), facteur de risque important à l'enfance pour le développement ultérieur de comportements délinquants et syndrome pour lequel les études de jumeaux indiquaient une héritabilité élevée (soit entre 60 et $90 \%$ selon les études; Thapar et al.,1999; Nadder et al., 2001), a été associé avec constance à différents marqueurs génétiques (DRD4, DAT-1, DRD5) liés au système dopaminergique (Bobb et al., 2004; Inserm, 2005; Rutter, 2006). De plus, malgré des résultats divergents entre les études, certains marqueurs génétiques liés aux transporteurs ou aux récepteurs de la dopamine et de la sérotonine ont été associés au comportement agressif et impulsif (Lesch, 2003 ; Pérusse et Gendreau, 2005; Pihl et Benkelfast, 2005 ; Rutter, 2006).

Si davantage d'études sont nécessaires pour identifier des marqueurs génétiques spécifiques associés aux comportements antisociaux, les résultats de ces recherches, comme ceux des études de jumeaux, excluent la possibilité d'une influence purement environnementale sur le développement de ces comportements. Enfin, les associations observées dans les études sur les neurotransmetteurs suggèrent que les gènes associés à ces systèmes cérébraux sont des candidats importants à des études de génétique moléculaire en lien avec le comportement antisocial. 


\section{Vers une étude intégrée des influences génétiques et environnementales}

Le fait d'étudier les comportements antisociaux en pouvant séparer les composantes de la variance de ces phénomènes en fonction du génotype ou de l'environnement d'un individu offre d'énormes possibilités. Lopportunité nouvelle de mener ces études s'inscrit dans le contexte d'un consensus international dans le domaine de la psychopathologie et de la psychiatrie développementales sur la nécessité d'explorer les phénomènes d'interaction entre les gènes et l'environnement pour la compréhension du développement et la mise au point de nouvelles approches thérapeutiques et préventives des troubles mentaux (Kandel, 1998; Rutter, 2002; Insel, 2003 ; Insel et Quirion, 2005 ; Moffitt et al., 2005). Or, ces conclusions proviennent particulièrement des recherches sur le comportement antisocial à travers le développement (Moffitt et al., 2005; Tremblay, 2006) et s'appliquent donc directement à la compréhension et au traitement de la délinquance. Les progrès récents en génomique et en technologies génétiques ont ouvert la porte à deux types d'études qui permettent véritablement pour la première fois de conduire des études épidémiologiques intégrant des facteurs génétiques et environnementaux spécifiques dans l'étude du comportement antisocial. Ces études sont certainement celles qui s'annoncent les plus prometteuses au cours des décennies à venir. Il s'agit des études d'interactions gènes-environnement et des études épigénétiques.

\section{Étude des interactions gène x/environnement}

Les études en génétique du comportement basées sur des échantillons de jumeaux ont non seulement rapporté avec constance une héritabilité importante pour le comportement antisocial (Arseneault et al., 2003) et différents troubles psychiatriques, dont les troubles de comportement chez les enfants (Nadder et al., 2001; Rhee et Waldman, 2002; Dick et al., 2005), mais elles ont également fait ressortir l'importance des phénomènes d'interaction gènes-environnement pour en comprendre le développement (Button et al., 2005 ; Jaffee et al., 2005). Ces résultats étaient cohérents avec ceux observés dans les études animales (Suomi, 2005b). Or, la possibilité nouvelle d'identifier l'ensemble des gènes et de cibler des marqueurs spécifiques à étudier en relation avec le comportement a entraîné une variété d'études vérifiant des hypothèses 
spécifiques de lien entre certains marqueurs génétiques et certaines dimensions-clés de l'environnement pour comprendre le développement de comportements particuliers, dont le comportement antisocial. Des études récentes ont examiné ce type d'interactions en utilisant des marqueurs génétiques associés aux neurotransmetteurs et ont rapporté des résultats significatifs, notamment pour des gènes liés aux systèmes dopaminergique et sérotoninergique (Caspi et al., 2002; 2003 ; Inserm, 2005).

L'étude de Caspi et collaborateurs dans la prestigieuse revue Science en 2002 a marqué le début de ce type d'investigation à grande échelle pour plusieurs groupes de recherche dans le monde. Ces chercheurs ont testé le génotype pour un polymorphisme de répétition (VNTR) du promoteur de la monoamine oxydase A (MAO-A) chez 499 garçons participant à une étude longitudinale et n'ont pas retrouvé d'association directe entre le gène et le développement de comportements antisociaux, mais ont observé une interaction avec la maltraitance des enfants: les enfants qui possédaient l'allèle court, indiquant une plus faible activité enzymatique, étaient plus à risque de présenter ces comportements lorsque maltraités, en comparaison avec leurs pairs possédant l'allèle long. Ces résultats étaient concluants pour quatre phénotypes associés au comportement antisocial, basés sur différentes sources d'information, à différentes périodes du développement: le diagnostic psychiatrique de troubles de la conduite à l'adolescence, le dossier criminel officiel, les traits de personnalité agressive et les symptômes de personnalité antisociale à l'âge adulte. De plus, il est important de noter que ces résultats ont été reproduits dans une autre étude longitudinale examinant le développement du trouble de la conduite en lien avec des conditions familiales défavorables (violence, négligence et discipline incohérente) et le polymorphisme de la MAO-A (Foley et al., 2004). Les études de Caspi, Foley et leurs collaborateurs suggèrent donc une vulnérabilité génétique chez certains individus à développer des comportements antisociaux, mais uniquement en présence de certaines conditions environnementales. Inversement, ils indiquent qu'un facteur de risque reconnu dans l'environnement des enfants pour le développement du comportement antisocial, soit la maltraitance, pourrait avoir un effet plus sévère chez les jeunes présentant un polymorphisme génétique particulier.

Une étude récente illustre un autre type d'étude d'interaction, cette fois entre un marqueur génétique et deux comportements observés, 
pouvant sous-tendre une influence différentielle de l'environnement selon le génotype d'un individu. DeYoung et ses collaborateurs (2006) ont observé un effet modérateur du gène $\mathrm{D} 4$, lié au récepteur de la dopamine, sur la relation entre les troubles externalisés (agressivité, opposition et hyperactivité) et les habiletés cognitives des garçons. Plus spécifiquement, la relation entre ces deux dimensions n'était observée que chez les individus ne possédant pas l'allèle-7, alors qu'elle n'était pas significative pour ceux en possédant au moins une copie. Ces résultats sont importants entre autres parce qu'ils représentent une hypothèse explicative de la variation observée à travers les études sur le lien entre la cognition et les troubles de comportement ou le comportement antisocial (Walsh, 2003). Ainsi, on peut faire l'hypothèse que chez certains individus, de faibles habiletés cognitives peuvent entraîner le développement de comportements antisociaux sous l'influence de leur environnement, alors que chez d'autres qui présentent un polymorphisme particulier, ce risque serait nul. En conséquence, chez ces derniers, si l'on devait intervenir pour diminuer le comportement antisocial, on peut supposer que le recours à une méthode cognitivo-comportementale, pourtant répandue dans les centres de traitement pour les délinquants (LeBlanc et al., 2000), serait moins efficace.

La convergence entre les résultats et les progrès récents de la recherche en criminologie clinique, en psychiatrie génétique, en épidémiologie psychiatrique et en génétique du comportement a donc ouvert une nouvelle perspective de recherche, soit l'étude des interactions gènesenvironnement basée sur des données génétiques moléculaires pour expliquer l'apparition et les trajectoires des troubles du comportement, des comportements antisociaux et des désordres psychiatriques. Les études futures vont poursuivre sur cette lancée afin de tester différents modèles d'interaction gènes-environnement, dont l'hypothèse d'une influence différentielle de certaines conditions environnementales (familiales ou sociales) selon le génotype de l'enfant (Caspi et al., 2002; 2003 ; Moffitt et al., 2005). Elles pourront également tester différents modèles d'association, par exemple, l'hypothèse que les pratiques parentales sont un médiateur de l'influence génétique du parent sur l'enfant. Les études de Carbonneau et collaborateurs (2002a; 2002b), à partir d'un échantillon de jumeaux âgés entre 8 et 16 ans, ont en effet démontré une base génétique commune aux pratiques parentales et aux comportements antisociaux des enfants et des adolescents. Ces résultats ont aussi été reproduits avec un échantillon de jumeaux québécois au 
préscolaire (Boivin et al., 2005). D'autres études s'intéresseront à l'hypothèse que le comportement parental constitue plutôt un modérateur venant amplifier ou diminuer une influence génétique plus directe (interaction gène-environnement, Rutter, 2002; Moffitt et al., 2005). Ces différentes études sont particulièrement prometteuses pour l'identification des enfants à risque de même que des dimensions à cibler pour d'éventuelles interventions.

\section{De l'environnement à l'organisation biologique:} les études épigénétiques

Une des pistes les plus récentes, mais également des plus excitantes pour la recherche à venir sur la relation entre les dimensions environnementales et génétiques, est certainement l'hypothèse épigénétique. Le terme épigénétique réfère aux mécanismes qui altèrent l'expression génétique sans modifier les gènes eux-mêmes (Weinhold, 2006). Les travaux pionniers de Meaney et ses collaborateurs (Meaney, 2001; Champagne et al., 2003; Weaver et al., 2004), qui observent un lien entre les comportements maternels et la méthylation des gènes en relation avec le comportement chez les rats, sont à cet égard fascinants. Ces chercheurs ont d'abord observé des variations dans le comportement maternel auprès des nouveau-nés au cours de la première semaine d'allaitement, certaines mères léchant davantage leurs petits. Or, cette différence de comportement maternel a par la suite été associée au développement de différences individuelles chez les rats sur le plan du comportement et de la réponse physiologique de l'axe HPA face au stress: les ratons davantage léchés par leur mère au moment de leur naissance étaient, une fois devenus adultes, moins peureux et présentaient une plus faible activité de l'axe HPA que les ratons dont la mère avait peu léché sa progéniture à la naissance. Les recherches ultérieures de ce groupe ont montré que ces différences sur le plan de la réaction au stress entre les rats devenus adultes étaient dues à une différence dans l'expression d'un gène promoteur de l'hippocampe, associé à la réponse endocrinienne et favorisant l'activité cérébrale au niveau du système sérotoninergique. Or, les observations réalisées ont indiqué que la différence entre les rats n'était pas attribuable à une différence sur le plan génétique, c'est-à-dire à une différence entre les gènes, mais plutôt à une différence sur le plan épigénétique, soit au niveau de l'expression d'un gène, présent chez les uns et les autres. Cette différence tenait au fait que les soins maternels 
au cours de la première semaine de vie avaient altéré de façon durable le niveau de méthylation de ce gène particulier, entraînant une réponse différente face au stress, même lorsque les rats étaient devenus adultes.

La méthylation est un processus biochimique qui affecte la retranscription du gène (Egger et al., 2004). En d'autres mots, sans modifier le génotype d'un individu, ce phénomène peut entraîner la suppression de l'activité de certains gènes. Les résultats de Meaney et ses collaborateurs suggèrent que la méthylation a un rôle important à jouer dans l'interaction entre l'environnement et l'expression génétique. Ainsi, il ne suffit pas d'avoir certaines caractéristiques génétiques, mais encore faut-il qu'elles soient activées, et cela pourrait bien être le résultat de l'interaction entre l'individu et son environnement. Meaney et ses collaborateurs ont ensuite administré aux rats un traitement sous forme de médication visant à contrebalancer l'effet de la méthylation. Leurs résultats montrent que la réponse des rats au stress a effectivement diminué. Il est important de noter, comme le soulignent les auteurs, que leurs observations concernent des variations comportementales normales à l'intérieur d'un environnement également normal, et donc qu'il ne s'agit pas de comportements extrêmes ou d'un environnement à risque. Les recherches de Meaney et ses collaborateurs ont soulevé un énorme intérêt dans la communauté scientifique (Rutter, 2005 ; 2006; Tremblay, 2006). Leur démonstration scientifique que l'environnement affecte la constitution génétique des individus en régulant l'expression de leurs gènes laisse entrevoir l'émergence d'un champ de recherche fascinant pour les décennies à venir: l'étude épigénétique du comportement humain. À cet égard, le comportement antisocial, phénotype à la croisée des disciplines de la criminologie clinique, de la psychopathologie développementale et de la psychiatrie, et pour lequel, comme nous l'avons vu, l'influence génétique a été démontrée, fera certainement l'objet de recherches intensives, étant donné l'importance également démontrée des facteurs environnementaux dans l'étiologie de ces comportements.

D'ailleurs, dans la foulée des résultats de Meaney et ses collaborateurs, Szyf et ses collègues (2007) ont entrepris une étude de méthylation en lien avec les comportements d'agressivité physique chez les garçons. Un sous-échantillon de l'Étude Longitudinale et Expérimentale de Montréal (ÉLEM) (Tremblay et al., 2003), 1037 garçons de milieu socioéconomique défavorisé, a été utilisé pour cette étude. Ces garçons avaient fait antérieurement l'objet d'une étude sur les trajectoires de 
comportements d'agressivité physique entre les âges de 6 et 15 ans (Nagin et Tremblay, 1999; 2001). Les résultats préliminaires, auprès de 16 sujets seulement, montrent une différence marquée entre les garçons cheminant sur une trajectoire d'agressivité chronique de l'enfance à l'adolescence et ceux qui suivent une trajectoire non agressive: les premiers présentent une plus grande méthylation au niveau de l'ADN total, de même que pour l'ADN des cellules $T$ du promoteur du gène ribosomal RNA (rRNA) (Szyf, 2007). Bien que ces résultats soient préliminaires et que cette étude soit toujours en cours, ils sont cohérents avec l'hypothèse que chez les humains, comme dans les modèles animaux, le développement des individus pourrait passer en partie par l'impact de l'environnement sur la méthylation et l'expression des gènes, en plus du génotype lui-même.

\section{Et l'intervention?}

Que représentent les résultats des études récentes qui viennent d'être abordées et les perspectives de recherche à venir sur le plan bio-psychosocial pour l'intervention future en criminologie clinique? Tout d'abord, il est évident que la pratique auprès des délinquants devra suivre le développement des connaissances en sciences biomédicales pour évoluer. Cette évolution devra passer à la fois par une meilleure identification des besoins du délinquant, selon son profil bio-psycho-social, et par le développement de méthodes intégrant les connaissances issues des recherches fondamentales (Pallone et Hennessy, 2000). En effet, il sera de plus en plus difficile d'ignorer ce que l'on sait maintenant et ce que l'on précisera manifestement davantage avec le temps sur les caractéristiques génétiques et neuropsychologiques des individus qui commettent des actes délinquants (Quadagno, 2003; Walsh et Ellis, 2003). Si cela se confirme avec les recherches futures, comment pourrons-nous offrir un traitement généralisé si on a les preuves scientifiques qu'il peut bénéficier à certains délinquants mais peu, ou pas, à d'autres? Et comment chercheurs et cliniciens pourront-ils tourner idéologiquement le dos au recours à la médication si l'on comprend maintenant que les comportements antisociaux doivent être considérés, au même titre que les problèmes de santé mentale auxquels ils sont fortement associés, comme des maladies du cerveau, comme cela est le cas avec l'épilepsie ou la schizophrénie, pour lesquelles un tel recours semble désormais aller de soi? 
Par ailleurs, comment s'intègreront les nouvelles connaissances issues des études en génétique du comportement? Déjà, depuis quelques années, les compagnies pharmaceutiques développent un nouveau champ de recherche, la pharmaco-génomique, dont le but est justement le développement de connaissances visant la création de médicaments destinés spécifiquement à correspondre aux génotypes des individus (Tremblay, 2005). La stratégie utilisée par le groupe de Meaney (Weaver et al., 2004) pour compenser l'effet de la méthylation sur l'expression génétique associée au stress chez les rats est un exemple de ce que pourraient être ces traitements. Bien que cette éventualité soit clairement envisagée chez les humains et que de tels traitements soient déjà appliqués avec succès au niveau expérimental dans le cas du cancer (Egger et al., 2004), nous ne sommes qu'au tout début de ce type de recherche. Il ne s'agit pas, ici, de promouvoir le recours à la médication comme méthode de choix pour intervenir auprès du délinquant dans le futur. D'ailleurs, la médication n'est pas la seule piste étudiée puisque les recherches en nutrition s'intéressent également au même type d'effet sur l'expression génétique dans la recherche sur le cancer (Weinhold, 2006). Il s'agit plutôt de reconnaître, sur la base de données scientifiques solides, que le comportement déviant et antisocial, tout comme les problèmes de santé mentale, sont le résultat d'une organisation biopsycho-sociale particulière, découlant elle-même de l'interaction entre une constitution génétique et un environnement psychosocial spécifiques d'un individu. En conséquence, le traitement qui leur sera offert devra, dans la mesure du possible, refléter cette réalité, ce que les recherches récentes et celles que l'on anticipe pour les années à venir permettent d'entrevoir.

Par ailleurs, les connaissances acquises au cours des dernières décennies de recherche sur le comportement délinquant soutiennent sans équivoque la nécessité d'adopter une perspective développementale dans nos efforts d'intervention visant à réduire ce phénomène et ses conséquences chez les individus (Inserm, 2005; Rutter et al., 2006; Tremblay, 2006). L'influence à long terme démontrée des facteurs périnataux et familiaux au cours des premières années de vie des enfants (Raine, 2002; Brennan et al., 2003), l'impact de ces facteurs sur l'organisation biologique des individus que suggèrent les études animales (Meaney, 2001; Weaver et al., 2004), l'apport démontré sur le développement cérébral de facteurs génétiques dont l'effet survient sans aucun doute tôt dans la vie des enfants et, en particulier, au cours de la période 
intra-utérine (Inserm, 2005 ; Rutter, 2006; Tremblay, 2006), et l'identification de facteurs de risque environnementaux à différentes périodes de la vie des individus (Farrington, 1994) convergent à soutenir une approche interventionnelle couvrant l'ensemble du développement. Les résultats de ces différentes études soutiennent également le mouvement amorcé dans la communauté scientifique et clinique, il y a une vingtaine d'années, pour promouvoir une stratégie préventive plutôt que thérapeutique (Tremblay et Craig, 1995; Farrington et Coid, 2003) et éclairent davantage le choix de société à faire quant à l'allocation des ressources d'aide aux différentes périodes du développement. Les effets à long terme des facteurs observables tôt dans la vie des enfants sont trop importants pour attendre que des diagnostics soient émis ou que des actes criminels soient commis avant d'intervenir (Fishbein, 2000). De surcroît, les études épidémiologiques en psychopathologie développementale et les études de trajectoires ont largement démontré que ce qui survient à l'adolescence comme problèmes de santé mentale ou comme comportement déviants était souvent observable bien plus tôt dans le développement, en particulier pour les cas chroniques (Costello et al., 2003 ; 2005 ; Kessler et al., 2006). Les travaux de Tremblay et collaborateurs sur l'agressivité physique (Nagin et Tremblay, 1999; Tremblay et al., 2004; Côté et al., 2006) sont éloquents sur ce plan. Plutôt que d'attendre qu'un comportement évolue vers un problème de santé mentale ou de délinquance, mieux vaut intervenir tôt pour prévenir, ou éduquer, pour soutenir les individus et les populations vulnérables, plutôt que de tenter de traiter plus tard, avec les coûts et les succès mitigés que l'on connaît (Lipsey et Wilson, 1998; Connor, 2002).

Bien sûr, l'idée d'adopter une approche développementale et biopsycho-sociale en intervenant tôt dans le développement et en abordant le travail clinique sur la base de considérations génétiques et pharmacologiques soulève d'emblée, et avec raison, de sérieuses préoccupations sur le plan de l'éthique. Les inquiétudes soulevées au cours des dernières décennies au sujet de la confidentialité et de la vie privée, du risque d'étiquetage ou de celui relié aux traitements pharmacologiques ont donné lieu à des procédures d'évaluation des pratiques et à des règles d'éthique, de déontologie et de droit qui protègent efficacement les individus tant sur le plan de l'intervention que de la recherche clinique. Le recours aux données génétiques, plus récent, a alimenté de nombreux débats, entre autres sur l'impact des marqueurs biologiques et sur le fait 
que, de par leur nature même, l'identification d'agents génétiques causals a des conséquences qui dépassent la personne et qui peuvent concerner à la fois sa famille, son groupe d'appartenance ou certaines parties de la population (Comité consultatif canadien de la biotechnologie, 2004). Le danger de stigmatisation individuelle ou collective est alors bien réel, comme celui de l'utilisation de l'information génétique au désavantage des individus ou des groupes. Or, si la recherche précède souvent les pratiques pour le développement des connaissances, on peut certes anticiper qu'il en soit de même sur le plan de l'éthique reliée à l'étude des facteurs biologiques et à leur intégration subséquente aux interventions cliniques. Par exemple, à l'issu d'une démarche de réflexion concertée, les trois organismes de recherche principaux qui subventionnent la recherche auprès des êtres humains au Canada, les Instituts de recherches en santé du Canada (IRSC), le Conseil de recherches en sciences naturelles et en génie du Canada (CRSNG) et le Conseil de recherches en sciences humaines du Canada (CRSH), ont adopté une politique commune qui définit des normes éthiques rigoureuses, notamment sur le plan de la recherche en génétique humaine (IRSC, CRSNG, CRSH, 1998). L'évolution des pratiques par l'intégration des connaissances biomédicales suit déjà le même chemin, comme cela est observé dans les règles, procédures et codes déontologiques qui régissent l'intervention en psychologie et en médecine. De plus, si l'interdisciplinarité et le travail multidisciplinaire sont déjà favorisés en criminologie clinique, l'évolution des connaissances biomédicales et leur intégration dans un cadre de pratique développemental va nécessiter de façon plus marquée encore la collaboration de spécialistes qui s'intéressent à différents aspects de l'individu et à différentes périodes de son développement, favorisant du même coup le transfert des normes d'éthique. Enfin, chercheurs et cliniciens ont également la responsabilité d'éduquer la population, pour qui l'influence génétique est généralement synonyme de déterminisme et les gènes souvent perçus par opposition à l'environnement lorsqu'il est question d'étiologie (Condit, 2007). Une diffusion responsable de l'information relative aux résultats de la recherche et aux innovations sur le plan clinique sensibilisera la population et diminuera d'autant les préjugés basés sur des perceptions erronées. 


\section{Conclusion}

En quoi la criminologie clinique des décennies à venir se différencierat-elle de ce que nous avons connu au cours du $\mathrm{xx}^{\mathrm{e}}$ siècle? Les résultats fascinants d'études multidisciplinaires récentes suggèrent que la compréhension et le traitement de la délinquance et de la criminalité, comme celle des maladies mentales, sont à l'aube de changements importants (Collins, 2004; Insel et Quirion, 2005). D'abord, un consensus émerge maintenant dans la communauté scientifique, selon lequel les comportements délinquants sont le résultat de causes multiples et complexes qu'un modèle théorique simple ou unidimensionnel ne peut expliquer (Walsh et Ellis, 2003 ; Carey et Gottesman, 2006; Rutter et al., 2006; Tremblay, 2006). Nous sommes optimistes de voir, à la lumière des connaissances issues de la recherche, les antagonismes primaires entre paradigmes céder la place à des modèles intégrant les différentes dimensions qui caractérisent un individu. Le réductionnisme, qu'il soit biologique, psychologique, environnemental ou social, va à l'encontre des conclusions de la recherche scientifique multidisciplinaire des dernières années (Cairns, 1996; Pallone et Hennessy, 2000; Lahey et al., 2003 ; Stoff et Susman, 2005b). Au contraire, la perspective biopsycho-sociale s'impose à la lumière des dernières décennies de recherche portant sur le comportement antisocial. Les progrès technologiques aidant, nous sommes maintenant en mesure de vérifier des hypothèses précises sur l'influence de facteurs génétiques ou biologiques spécifiques et de modéliser, à l'intérieur d'une même analyse, les interactions de ces facteurs avec certaines dimensions qui agissent à différents niveaux de l'environnement familial ou social et à différentes périodes du développement (Moffitt et al., 2005). Étant donné la nature même de ces différents facteurs et les connaissances issues des études longitudinales qui montrent l'évolution du phénomène de la délinquance à travers l'ensemble du développement, il semble incontournable qu'un cadre développemental soit la base de la recherche et de l'intervention en criminologie clinique.

On estime que de 50 à $70 \%$ des crimes sont commis par près de $6 \%$ de délinquants récidivistes. Cette dernière constatation ressort de plusieurs études menées auprès de populations générales tant au Canada qu'à travers le monde (Tremblay, 2000). Selon le modèle de Moffitt (1993), soutenu par plusieurs études et trouvant écho dans la définition des troubles de la conduite du DSM (APA, 2000), ces délinquants 
seraient ceux dont les comportements antisociaux ont débuté précocement, soit avant l'âge de dix ans. Cette hypothèse est cohérente avec le résultat des études sur les trajectoires des comportements antisociaux et avec ceux des études en épidémiologie psychiatrique, qui convergent à identifier les premières manifestations des troubles de comportement dès les premières années de vie des enfants (Gadow et al., 2001; Keenan et Wakchlag, 2004; Tremblay et al., 2004; Egger et al., 2006; Romano et al., 2006). Ces différents résultats sur la description du phénomène du comportement antisocial et sur son développement sont également cohérents avec l'hypothèse de facteurs génétiques multiples, qui influenceraient ce comportement tôt dans la vie des enfants par le biais de facteurs neurobiologiques sous-jacents (Raine, 1993; Brennan et al., 2003; Mattson, 2003).

Comme nous l'avons vu également, l'influence de l'environnement familial et social est incontournable pour le développement d'un individu vers une trajectoire déviante: quelle que soit la constitution génétique de ce dernier, aucun marqueur ou facteur de risque individuel n'apparaît suffisant sans un apport spécifique de son environnement. Mais, inversement, les études récentes et le courant de recherche qui s'active en sciences biomédicales indiquent qu'intervenir sur l'environnement sans tenir compte des facteurs biologiques irait à l'encontre de connaissances établies et des principes mêmes de l'intervention clinique en criminologie: offrir une intervention différentielle répondant aux besoins particuliers des individus dans le but d'avoir une plus grande efficacité (LeBlanc et al., 2000; Pallone et Hennessy, 2000; Connor, 2002). Et si les préoccupations éthiques commandent avec raison la prudence et un encadrement rigoureux dans le recours à des stratégies basées en partie sur ces facteurs, il serait tout aussi contre l'éthique d'ignorer des connaissances scientifiques pouvant améliorer la vie des individus (Comings, 2000). Le siècle qui vient sera sans aucun doute le siècle de la génétique et des neurosciences dans la recherche sur le comportement humain, et l'avenir des disciplines comme la criminologie, qui impliquent une intervention auprès des individus pour traiter ou prévenir un comportement, passe certainement par l'intégration des connaissances biomédicales aux pratiques psychosociales établies, à travers le développement d'une stratégie de recherche et d'une approche clinique véritablement bio-psycho-sociale (Ellis, 2003). Si l'avènement de ce type de recherche et son impact sur la compréhension du comportement antisocial sont récents, les possibilités sans précédent qui 
s'offrent maintenant aux chercheurs et aux intervenants en criminologie clinique rendent la poursuite active de cette nouvelle stratégie incontournable.

Les défis à venir sont toutefois nombreux. En effet, l'identification des marqueurs génétiques importants, des processus neuropsychiatriques, de même que la caractérisation et la quantification des facteurs environnementaux liés au comportement antisocial est un prélude aux études d'interaction gènes-environnement dans une perspective interventionniste, et cela nécessitera bon nombre d'études. Mais aussi, les interactions entre les gènes eux-mêmes et entre les facteurs environnementaux demeurent des sources potentielles et probables de variation dans l'étiologie du comportement délinquant (Rutter, 2002) et leur compréhension impliquera assurément une variété et une quantité de recherches importantes. Par ailleurs, comme nous l'avons souligné, malgré des années de recherche, la définition de phénotypes ou de comportements spécifiques dans le champ de la criminologie clinique, basés sur une perspective longitudinale plutôt que sur un seul point de mesure dans le temps, demeure incomplète et devra compter sur l'apport de recherches ultérieures. Il s'agit d'une étape cruciale pour la réalisation d'études génétiques et épigénétiques. Comme nous l'avons vu, les études sur les endophénotypes devraient contribuer à cette démarche d'identification ou de distinction phénotypique (Barker et al., 2007). À cet égard, une problématique importante qui devra être abordée concerne la distinction entre les variations à l'intérieur d'un comportement considéré comme normal et celles qui indiquent une pathologie ou un problème d'adaptation psychosocial. L'agressivité constitue un bon exemple de comportement associé à la délinquance, mais faisant à la base intrinsèquement partie du répertoire humain (Tremblay et al., 2005).

Plus que jamais, on peut considérer que les efforts de recherche examinant le comportement criminel en sciences sociales et en sciences biomédicales devront intégrer leurs perspectives et leurs stratégies, si l'on aspire sérieusement à faire évoluer nos connaissances et notre capacité d'intervenir auprès des délinquants au-delà de ce que l'évolution, largement parallèle, de ces disciplines au cours des dernières décennies, nous a amenés. D'aucuns considèrent que les résultats des interventions en sciences sociales pour réduire la délinquance vont demeurer limités si de telles interventions ne tiennent pas compte des connaissances issues de la recherche biomédicale (Pallone et Hennessy, 
2000). Il faut admettre qu'à ce jour les résultats des traitements auprès des délinquants demeurent mitigés (Lipsey et Wilson, 1998; Connor, 2002). Mais il est aussi vrai que la compréhension du comportement psychosocial et de l'environnement familial et social est indispensable aux chercheurs en neurosciences et en génétique, pour progresser vers les études des mécanismes neurobiologiques, les études d'interaction gènes-environnement et les études épigénétiques nécessaires à l'évolution de ces domaines en lien avec la santé mentale et le comportement antisocial. À ce titre, à l'aube du xxI siècle, la collaboration entre les disciplines représente la meilleure stratégie pour l'avancement de la recherche et de l'intervention en criminologie clinique, comme elle l'est pour toute discipline qui s'intéresse au comportement humain.

\section{Références}

Adler, F. A., Mueller, G. O. W., \& Laufer, W. S. (1995). Criminology. New York: McGraw-Hill.

Akers, R. L. (1994). Criminological theories: Introduction and evaluation. Los Angeles: Roxbury publishing company.

American Psychiatric Association (2000). Diagnostic and Statistical Manual of Mental Disorders, Fourth edition. Text Revision. Washington, DC: American Psychiatric Association.

Andrews, D. A., \& Bonta, J. (2003). The psychology of criminal conduct ( $3^{\text {rd }}$ edition). Cincinnati, $\mathrm{OH}$ : Anderson publishing Co.

Arseneault, L., Moffitt, T. E., Caspi, A., Taylor, P. J., \& Silva, P. A. (2000). Mental disorders and violence in a total birth cohort. Archives of general psychiatry, 57, 979-986.

Arseneault, L., Tremblay, R. E., Boulerice, B., \& Saucier, J.-F. (2002). Obstetrical complications and violent delinquency: Testing two developmental pathways. Child Development, 73 (2), 496-508.

Arseneault, L., Moffitt, T. E., Caspi, A., Taylor, A., Rijsdijk F. V., Jaffee S. R., Ablow J. C., \& Measelle, J. R. (2003). Strong genetic effects on cross-situational antisocial behaviour among 5-year-old children according to mothers, teachers, examiner-observers, and twins' self-reports. Journal of Child Psychology and Psychiatry, 44 (6), 832-848.

Barker, E. D., Séguin, J. R., White, H. R., Bates, M. E., Lacourse, É., Carbonneau, R., \& Tremblay, R. E. (2007). Developmental trajectories of male physical violence and theft: Relation to neurocognitive performance. Archives of General Psychiatry, 64, 592-599.

Beitchman J. H., Baldassarra, L., Mik, H., De Luca V., King N., Bender D., Ehtesham S., \& Kennedy, J. L. (2006). Serotonin transporter polymorphisms 
and persistent, pervasive childhood aggression. American Journal of Psychiatry, 163 (6), 1103-1105.

Berman, M. E., \& Coccaro, E. F. (1998). Neurobiologic correlates of violence: relevance to criminal responsibility. Behaviour Sciences and the Law, 16, 303318.

Blumensohn, R., Ratzoni, G., \& Weitzman, A. (1995). Reduction in serotonin 5 HT2 receptor binding on platelets of delinquent adolescents. Psychopharmacology, 188, 354-356.

Bobb, A. J., Castellanos, F. X., Addington, A. M., \& Rapoport, J. L. (2004). Molecular genetic studies of ADHD: 1991 to 2004. American Journal of Medical Genetics Part B : Neuropsychiatric Genetics, 141B (6), 551-565.

Boivin, M., Pérusse, D., Dionne, G., Saysset, V., Zoccolillo, M., Tarabulsy, G. M., Tremblay, N., \& Tremblay, R. E. (2005). The genetic-environmental etiology of parent's perceptions and self-assessed behaviours toward their 5 -months-old infants in a large twin and singleton sample. Journal of Child Psychology and Psychiatry, 46 (6), 612-630.

Brennan, P. A., Grekin, E. R., \& Mednick S. A. (2003). Prenatal and perinatal influences on conduct disorder and serious delinquency. In B. B. Lahey, T. E. Moffitt \& A. Caspi (eds.), Causes of conduct disorder and juvenile delinquency (319-344). New York: Guilford press.

Broidy, L. M., Nagin, D. S., Tremblay, R. E., Bates, J. E., Brame, B., Dodge, K., Fergusson, D., Horwood, J., Loeber, R., Laird, R., Lynam, D., Moffitt, T., Pettit, G. S., \& Vitaro, F. (2003). Developmental trajectories of childhood disruptive behaviors and adolescent delinquency: A six site, cross-national study. Developmental Psychology, 39 (20), 222-245.

Brown, T. G., Gianoulakis, C., Tremblay, J., Nadeau, L., Dongier, M., Ng Ying Kin, N. M. K., Seraganian, P., \& Ouimet, M. C. (2005). Salivary cortisol: a predictor of convictions for driving under the influence of alcohol? Alcobol and Alcobolism, 40 (5), 474-481.

Brown, G. L., Goodwin, F. K., Ballenger, J. C., Goyer, P. F., \& Major, L. F. (1979). Aggression in humans correlates with cerebrospinal fluid amine metabolites. Psychiatry Research, 1, 131-139.

Button, T. M., Scourfield, J., Martin, N., Purcell, S., \& McGuffin, P. (2005). Family dysfunction interacts with genes in the causation of antisocial symptoms. Behavior Genetics, 35 (2), 115-20.

Cairns, R. B. (1996). Aggression from a developmental perspective: Genes, environments and interactions. In G. R. Block \& J. A. Goode (eds.), Genetics of criminal and antisocial behaviour (45-60). Chichester, UK: Wiley.

Carbonneau, R. (2002). Developmental trajectories leading to delinquency and substance use in adolescence: Results from Quebec studies. In S. Brochu, C. DaAgra \& M.-M. Cousineau (eds.), Drugs and crime deviant pathways (8596). London: Ashgate.

Carbonneau, R., Eaves, L.J., Silberg, J. L., Hewitt, J. K., Simonoff, E., \& Rutter, M. (2002a). Assessment of the within family environment in twins: Absolute versus differential ratings, and relationship with Conduct problems. Journal of Child Psychology and Psychiatry, 43, 1-11. 
Carbonneau, R., Rutter, M., Silberg, J. L., Simonoff, E., Hewitt, J. K., \& Eaves, L. J. (2002b). Assessment of genetic and environmental influences on differential ratings of within family experiences and relationships in twins. Psychological Medicine, 32, 729-741.

Carbonneau, R., Fontaine, N., Vitaro, F., \& Tremblay, R. E. (2006: 23-27 octobre). Trajectoires de développement de l'byperactivité au préscolaire et adaptation des enfants au moment de l'entrée à l'école. $10^{\text {es }}$ Journées annuelles de santé publique (JASP). Montréal, Québec, Canada.

Carey, G. C., \& Gottesman, I. I. (2006). Genes and antisocial behaviour: Perceived versus real threats to jurisprudence. Journal of Law, Medicine and Ethics, 34 (2), 342-351.

Caspi, A., McClay, J., Moffitt, T. E., Mill, J., Martin, J., Craig, I. W., Taylor, A., $\&$ Poulton, R. (2002). Role of genotype in the cycle of violence in maltreated children. Science, 297 (5582), 851-854.

Caspi, A., Sugden, K., Moffitt, T. E., Taylor, A., Craig, I. W., Harrington, H., McClay, J., Mill, J., Martin, J., Braithwaite, A., \& Poulton, R. (2003). Influence of life stress on depression: Moderation by a polymorphism in the 5-HTT gene. Science, 301 (5631), 386-389.

Champagne, F. A., Francis, D. D., Mar, A., \& Meaney, M. J. (2003). Variations in maternal care in the rat as a mediating influence for the effects of environment on development. Physiology \& Behavior, 79, 359-371.

Clark, W. R., \& Grunstein, M. (2000). Are we hardwired? The role of genes in buman behaviour. Oxford: Oxford University Press.

Coccaro, E. F. (1998). Central neurotransmitter function in human aggression and impulsivity. In M. Maes \& E. Coccaro (eds.), Neurobiology and clinical views on aggression and impulsivity (143-168). New York: Wiley.

Coccaro, E. F., Kavoussi, R. J., \& McNamee, B. (2000). Central neurotransmitter function in criminal aggression. In D. H. Fishbein (Ed.), The science, treatment, and prevention of antisocial behaviours (chap. 6, 1-16). Kingston, N-J: Civic Research Institute.

Collins, R. E. (2004). Onset and Desistance in Criminal Careers : Neurobiology and the Age-Crime Relationship. Journal of Offender Rebabilitation, 39 (3), $1-19$.

Comings, D. E. (2000). The role of genetics in ADHD and conduct disorder - relevance to the treatment of recidivistic antisocial behaviour. In D. H. Fishbein (ed.), The science, treatment, and prevention of antisocial behaviours (chap. 16, 1-25). Kingston, N-J: Civic Research Institute.

Comité consultatif canadien de la biotechnologie (2004). La recherche génétique et la protection de la vie privée. Mémoire consultatif. Ottawa: Gouvernement du Canada.

Condit, C. M. (2007). How geneticists can help reporters to get their story right. Nature Reviews-Genetics, 8, 815-820.

Connor, D. F. (2002). Aggression $\mathcal{W}$ antisocial behaviour in children and adolescents: Research and Treatment. New York: Guilford press. 
Costello, J. E., Mustillo, S., Erkanli, A., Keeler, G., \& Angold, A. (2003). Prevalence and development of psychiatric disorders in childhood and adolescence. Archives of General Psychiatry, 60, 837-844.

Costello, J. E., Egger, H., \& Angold, A. (2005). 10-year research update review: The epidemiology of child and adolescent psychiatric disorders: I. Methods and public health burden. Journal of the American Academy of Child and Adolescent Psychiatry, 44 (10), 972-986.

Côté, S., Vaillancourt, T., LeBlanc, J. C., Nagin, D. S., \& Tremblay, R. E. (2006). The development of physical aggression from toddlerhood to pre-adolescence: A nation wide longitudinal study of Canadian children. Journal of Abnormal Child Psychology, 34 (1), 71-85.

Davidson, R. J., Putnam, K. M., \& Larson, C. L. (2000). Dysfunction in the neural circuitry of emotion regulation - a possible prelude to violence. Science, 289, 591-594.

DeYoung, C. G., Peterson, J. B., Séguin, J. R., Mejia, J. M., Pihl, R. O., Beitchman, J. H., Jain, U., Tremblay, R. E., Kennedy, J. L., \& Palmour, R. M. (2006). The dopamine D4 receptor gene and moderation of the association between externalizing behavior and IQ. Archives of General Psychiatry, 63 (12), 1410-1416.

Dick, D. M., Viken, R. J., Kaprio, J., Pulkinen, L., \& Rose, R. J. (2005). Understanding the covariation among childhood externalizing symptoms: Genetic and environmental influences on Conduct Disorder, Attention Deficit Hyperactivity Disorder, and Oppositional Defiant Disorder symptoms. Journal of Abnormal Child Psychology, 33 (2), 219-229.

Egger, G., Liang, G., Aparicio, A., \& Jones, P. A. (2004). Epigenetics in human disease and prospects for epigenetic therapy. Nature, 429, 457-463.

Egger, H. L., \& Angold, A. (2006). Common emotional and behavioural disorders in preschool children: presentation, nosology, and epidemiology. Journal of Child Psychology and Psychiatry, 47 (3), 313-337.

Eklund, J., Alm, P. O., \& Klinteberg, B. A. (2005). Monoamine oxidase activity and tri-iodothyronine level in violent offenders with early behavioural problems. Neuropsychobiology, 52, 122-129.

Eley, T. C., Lichenstein, P., \& Stevenson, J. (1999). Sex differences in the etiology of aggressive and nonaggressive behaviour: Results from two twin studies. Child Development, 70 (1), 155-168.

Ellis, L. (2003). So you want to be a biosocial criminologist? Advice from the underground. In A. Walsh \& L. Ellis (eds.), Biosocial criminology: Challenging environmentalism's supremacy (249-256). New York: Nova Science Publishers.

Farrington, D. P. (1994). Childhood, adolescent, and adult features of violent males. In L. R. Huesman (ed.), Aggressive behavior: Current perspectives (215240). New York: Plenum Press.

Farrington, D. P. (2002). Families and crime. In J. Q. Wilson \& J. P. Petersilia (eds.), Crime: Public policies for crime control ( $2^{\text {nd }}$ ed., 129-148). Oakland, CA: Institute for contemporary studies press.

Farrington, D. P., \& Coid, J. W. (2003). Early prevention of antisocial behaviour. Cambridge: Cambridge University Press. 
Fergusson, D. M., Horwood, L. J., \& Ridder, E. M. (2004). Show me the child at seven: The consequences of conduct problems in childhood for psychosocial functioning in adulthood. Journal of Child Psychology and Psychiatry, $45,1-13$.

Filley, C. M., Price, B. H., Nell, V. D., Antoinette, T., Morgan, A., Bresnahan, J., Pincus, J., Gelbort, M., Weissberg, M., \& Kelly, J. (2001). Toward an understanding of violence: Neurobehavioral aspects of unwarranted physical aggression: Aspen neurobehavioral conference consensus statement. Neuropsychiatry, Neuropsychology and Behavioral Neurology, 14, 1-14.

Fishbein, D. H. (2000). How can neurobiological research inform prevention strategies. In D. H. Fishbein (ed.), The science, treatment, and prevention of antisocial behaviors (chap. 25, 1-30). Kingston, N-J: Civic Research Institute.

Fishbein, D. H. (2001). Biobehavioral perspectives in criminology. Belmont, CA: Wadsworth/Thompson Learning.

Fishbein, D. H. (2003). Neuropsychological and emotional regulatory processes in antisocial behaviour. In A.Walsh \& L. Ellis (eds.), Biosocial criminology: Challenging environmentalism's supremacy (185-208). New York: Nova Science Publishers.

Fogel, B. S., Schiffer, R. B., \& Rau, S. M. (2000). Synopsis of psychiatry. New York: Lippincott, Williams and Wilkins.

Foley, D. L., Eaves, L. J., Wormley, B., Silberg, J., Maes, H. H., Kuhn, J., \& Riley, B. (2004). Childhood adversity, monoamine oxidase A genotype, and risk for conduct disorder. Archives of General Psychiatry, 61, 738-744.

Fontaine, N., Carbonneau, R., Barker, E. D., Vitaro, F., Hébert, M., Côté, S. M., Nagin, D. S., Zoccolillo, M., \& Tremblay, R. E. (sous presse). Girls' hyperactivity and physical aggression during childhood predict adjustment problems in early adulthood: A 15-year longitudinal study. Archives of General Psychiatry.

Gadow, K. D., Sprafkin, J., \& Nolan, E. E. (2001). DSM-IV symptoms in community and clinic preschool children. Journal of the American Academy of Child and Adolescent Psychiatry, 40 (12), 1383-1392.

Gendreau, P. L., \& Archer, J. (2005). Subtypes of aggression in humans and animals. In R. E. Tremblay, W. W. Hartup \& J. Archer (eds.), Developmental origins of aggression (25-46). New York: Guilford Press.

Giancola, P. R. (2000). Neuropsychological functioning and antisocial behaviour - implications for etiology and prevention. In D. H. Fishbein (ed.), The science, treatment, and prevention of antisocial behaviours (chap. 11, 1-16). Kingston, N-J: Civic Research Institute.

Glueck, S., \& Glueck, E. T. (1950). Unraveling juvenile delinquency. Cambridge, MA: Harvard University Press.

Gottesman, I., \& Goldsmith, H. H. (1994). Developmental psychopathology of antisocial behaviour: Inserting genes into its ontogenesis and epigenesist. In C. A. Nelson (ed.), Threats to optimal development: Integrating biological, psychological, and social risk factors (69-104). Hillsdale, N-J. : Erlbaum. 
Hirschfield, P., Maschi, T., White, R. H., Goldman-Traub, L., \& Loeber, R. (2006). Mental Health and juvenile arrests: Criminality, criminalization, or compassion? Criminology, 44 (3), 593-630.

Hodgins, S. (1992). Mental disorder, intellectual deficiency, and crime: Evidence from a birth cohort. Archives of General Psychiatry, 49, 476-483.

Hodgins, S. (2001). The major mental disorders and crime: Stop debating and start treating and preventing. International Journal of Law and Psychiatry, 24, 427-446.

Hodgins, S., \& Muller-Isberner, R. (2000). Violence, crime and mentally disordered offenders. New York: Wiley.

Huesman, L. R., Eron, L. D., Lefkowitz, M. M., \& Walder, L. O. (1984). Stability of aggression over time and generations. Developmental Psychology, 20, 1120-1134.

Insel, T. R. (2003). Psychiatry in the Genomics Era. American Journal of Psychiatry, 160, 616-620.

Insel, T. R., \& Quirion, R. (2005). Psychiatry as a clinical neuroscience discipline. Journal of the American Medical Association, 294, 2221-2224.

Inserm - Institut national de la santé et de la recherche médicale, expertise collective (2005). Trouble des conduites chez l'enfant et l'adolescent. Paris: Les éditions Inserm.

Ishikawa, S. S., \& Raine, A. (2003). Prefrontal deficits and antisocial behaviour: A causal model. In B. B. Lahey, T. E., Moffitt \& A. Caspi (eds.), Causes of conduct disorder and juvenile delinquency (277-304). New York: Guilford press.

Instituts de recherche en santé du Canada, Conseil de recherches en sciences naturelles et en génie du Canada, Conseil de recherches en sciences humaines du Canada (1998 [avec les modifications de 2000, 2002 et 2005]). Énoncé de politique des trois Conseils: Éthique de la recherche avec des êtres bumains, Ottawa: Travaux publics et Services gouvernementaux Canada.

Jaffee, S. R., Caspi, A., Moffitt, T. E., Dodge, K. A., Rutter, M., Taylor, A., \& Tully, L. A. (2005). Nature x nurture: Genetic vulnerabilities interact with physical maltreatment to promote conduct problems. Development and Psychopathology, 17, 67-84.

Kandel, E. R. (1998). A new intellectual framework for psychiatry. American Journal of Psychiatry, 155 (4), 457-469.

Keenan, K., \& Wakschlag, L. S. (2004). Are oppositional defiant and conduct disorder symptoms normative behaviors in preschoolers? A comparison of referred and nonreferred children. American Journal of Psychiatry, 161, 356358.

Keenan, K., \& Shaw, D. S. (2003). Starting at the beginning: Exploring the etiology of antisocial behaviour in the first years of life. In B. B. Lahey, T. E., Moffitt, \& A. Caspi (eds.), Causes of conduct disorder and juvenile delinquency (153-182). New York: Guilford press.

Kendler, K. S. (2005). "A gene for...": The nature of gene action in psychiatric disorders. American Journal of Psychiatry, 162, 1243-1252. 
Kessler, R. C., Berglund, P., Demler, O., Jin, R., Merikangas, K. R., \& Walters, E. E. (2006). Lifetime prevalence and age-of-onset distributions of DSM-IV disorders in the National Comorbidity Survey Replication. Archives of General Psychiatry, 62, 593-602.

Lahey, B. B., Moffitt, T. E., \& Caspi, A. (2003). Causes of conduct disorder and juvenile delinquency. New York: Guilford press.

Lavigne, J. V., Gibbons, R. D., Christoffel, K. K., Arend, R., Rosenbaum, D., Binns, H., Dawson, N., Sobel, H., \& Isaacs, C. (1996). Prevalence rates and correlates of psychiatric disorders among preschool children. Journal of the American Academy of Child and Adolescent Psychiatry, 35 (2), 204-214.

Le Protecteur du citoyen (2006). Rapport annuel 2005-2006. Assemblée nationale Québec. Québec: Ministère de la Sécurité publique.

LeBlanc, M., Dionne, J., Proulx, J., Grégoire, J. C., \& Trudeau-Le Blanc, P. (2000). Intervenir autrement: un modèle différentiel pour les adolescents en difficulté. Montréal: Presses de l'Université de Montréal.

Lesch, K. P. (2003). The serotonergic dimension of aggression and violence. In M. P. Mattson (ed.), Neurobiology of aggression: Understanding and preventing violence (33-64). Totowa, N-J: Humana press.

Lipsey, M., \& Wilson, D. B. (1998). Effective intervention for serious juvenile offenders: A synthesis of research. In R. Loeber \& D. P. Farrington (eds.), Serious $\mathfrak{F}$ violent juvenile offenders: Risk factors and successful interventions (313345). Thousand Oaks: Sage publications.

Loeber, R. (1996). Developmental continuity, change, and pathways in male juvenile problem behaviors and delinquency. In J. D. Hawkins (ed.), Delinquency and crime: Current theories (1-27). Cambridge: Cambridge University Press.

Mattson, M. P. (2003). Neurobiology of aggression: Understanding and preventing violence. Totowa, N-J: Humana press.

McCord, W., McCord, J., \& Zola, I. K. (1959). Origins of crime. New York: Columbia University Press.

Meaney, M. J. (2001). Maternal care, gene expression, and the transmission of individual differences in stress reactivity across generations. Annual Revue of Neuroscience, 24, 1161-1192.

Moffitt, T. E. (1993). Adolescence-limited and life-course persistent antisocial behavior: A developmental taxonomy. Psychological Review, 100 (4), 674701.

Moffitt, T. E. (2003) Life-course persistent and adolescence-limited antisocial behaviour: A 10-year research review and a research agenda. In B. B. Lahey, T. E. Moffitt \& A. Caspi (eds.), Causes of conduct disorder and juvenile delinquency (49-75). New York: Guilford press.

Moffitt, T. E., \& Walsh, A. (2003). The adolescence-limited/life-course persistent theory of antisocial behaviour: What have we learned? In A. Walsh \& L. Ellis (eds.), Biosocial criminology: Challenging environmentalism's supremacy (125-144). New York: Nova Science Publishers. 
Moffitt, T. E., Caspi, A., \& Rutter, M. (2005). Strategy for investigating interactions between measured genes and measured environments. Archives of General Psychiatry, 62, 473-481.

Muller-Isberner, R., \& Hodgins, S. (2000). Evidence-based treatment for mentally disordered offenders. In S. Hodgins \& R. Muller-Isberner (Eds.), Violence, crime and mentally disordered offenders (7-38). New York: Wiley.

Nadder, T. S., Rutter, M., Silberg, J. L., Maes, H. H., \& Eaves, L. J. (2002). Genetic effects on the variation and covariation of attention deficithyperactivity disorder (ADHD) and oppositional-defiant disorder/conduct disorder (ODD/CD) symptomatologies across informant and occasion of measurement. Psychological Medicine, 32, 39-53.

Nagin, D., \& Tremblay, R. E. (1999). Trajectories of boys' physical aggression, opposition, and hyperactivity on the path to physically violent and nonviolent juvenile delinquency. Child Development, 70 (5), 1181-1196.

Nagin, D., \& Tremblay, R. E. (2001). Parental and early childhood predictors of persistent physical aggression in boys from kindergarten to high school. Archives of General Psychiatry, 58, 389-394.

Nigg, J. T., \& Huang-Pollock, C. L. (2003). An early-onset model of the role of executive functions and intelligence in conduct disorder/delinquency. In B. B Lahey, T. E Moffitt \& A. Caspi (eds.), Causes of conduct disorder and juvenile delinquency (227-253). New York: Guilford press.

Pallone, N. J., \& Hennessy, J. J. (2000). Indifferent communication between social science and neuroscience - The case of "biological brain-proneness" for criminal aggression. In D. H. Fishbein (ed.), The science, treatment, and prevention of antisocial behaviors (chap. 22, 1-13). Kingston, N-J: Civic Research Institute.

Patterson, G. R. (1982). Coercive family process. Eugene, OR: Castalia Press.

Paus, T. (2005). Mapping brain development and aggression. In R. E. Tremblay, W. W. Hartup \& J. Archer (eds.), Developmental origins of aggression (242-260). New York: Guilford Press.

Pérusse, D., \& Gendreau, P. L. (2005). Genetics and the development of aggression. In R. E. Tremblay, W. W. Hartup \& J. Archer (eds.), Developmental origins of aggression (223-241). New York: Guilford Press.

Pihl, R. O., \& Benkelfast, C. (2005). Neuromodulators in the development and expression of inhibition and aggression. In R. E. Tremblay, W. W. Hartup $\&$ J. Archer (eds.), Developmental origins of aggression (261-280). New York: Guilford Press.

Quadagno, D. (2003). Genes, brains, hormones, and violence: Interactions within complex environments. In A. Walsh \& L. Ellis (eds.), Biosocial criminology: Challenging environmentalism's supremacy (167-184). New York: Nova Science Publishers.

Raine, A. (1993). The psychopathology of crime: Criminal behaviour as a clinical disorder. San Diego, CA: Academic Press.

Raine, A. (2002). Annotation: The role of prefrontal deficits, low autonomic arousal, and early health factors in the development of antisocial and aggres- 
sive behaviour in children. Journal of Child Psychology and Psychiatry, 43 (4), 417-434.

Raine, A. (2005). The interaction of biological and social measures in the explanation of antisocial and violent behavior. In D. M. Stoff \& E. J. Susman (eds.), Developmental psychobiology of Aggression (13-42). New York: Cambridge University Press.

Rhee, S. H., \& Waldman, I. D. (2002). Genetic and environmental influences on antisocial behavior: a meta-analysis of twin and adoption studies. Psychological Bulletin, 128, 490-529.

Romano, E., Tremblay, R. E., Farhat, A., \& Côté, S. (2006). Development and prediction of hyperactive symptoms from 2 to 7 years in a population-based sample. Pediatric, 117 (6), 2101-2110.

Rutter, M. (2002). The interplay of nature, nurture, and developmental influences: The challenge ahead for mental health. Archives of General Psychiatry, 59, 996-1000.

Rutter, M. (2005). How the environment affects mental health. British Journal of Psychiatry, 186, 4-6.

Rutter, M. (2006). Genes and behavior. Malden, MA: Blackwell publishing.

Rutter, M., Kim-Cohen, J., \& Maughan, B. (2006). Continuities and discontinuities in psychopathology between childhood and adult life. Journal of Child Psychology and Psychiatry, 47 (3/4), 276-295.

Seguin, J. R., \& Zelazo, P. D. (2005). Executive function in early physical aggression. In R. E. Tremblay, W. W. Hartup \& Archer, J. (eds.), Developmental origins of aggression (307-329). New York: Guilford Press.

Shaw, D. S., Lacourse, E., \& Nagin, D. S. (2005). Developmental trajectories of conduct problems and hyperactivity from ages 2 to 10. Journal of Child Psychology and Psychiatry, 46 (9), 931-942.

Shih, J. C., \& Thompson, R. F. (1999). Psychiatric genetics '99: Monoamine oxydase in neuropsychiatry and behaviour. American Journal of Human Genetics, 65, 593-598.

Shoemaker, D. J. (2000). Theories of delinquency: An examination of explanations of delinquent behaviour ( $4^{\mathrm{e}}$ ed.). Oxford: Oxford University Press.

Siever, L., \& Trestman, R. L. (1993). The serotonin system and aggressive personality disorder. International Clinical Pharmacology, 8, 33-39.

Stattin, H., \& Magnusson, D. (1989). The role of early aggressive behavior in the frequency, seriousness, and types of later crime. Journal of Consulting and Clinical Psychology, 57, 710-718.

Stoff, D. M., \& Susman, E. J. (2005a). Developmental psychobiology of Aggression. New York: Cambridge University Press.

Stoff, D. M., \& Susman, E. J. (2005b). Integrated perspective for psychobiological research in aggression: An introduction. In D. M. Stoff \& E. J. Susman (eds.), Developmental psychobiology of Aggression (3-10). New York: Cambridge University Press.

Suomi, S. J. (2003). Social and biological mechanisms underlying impulsive aggressiveness in rhesus monkeys. In B. B. Lahey, T. E. Moffitt \& A. Caspi 
(eds.), Causes of conduct disorder and juvenile delinquency (345-362). New York: Guilford press.

Suomi, S. J. (2005a). Genetic and environmental factors influencing the expression of impulsive aggression and serotonergic functioning in rhesus monkeys. In R. E. Tremblay, W. W. Hartup, \& J. Archer (eds.), Developmental origins of aggression (63-82). New York: Guilford Press.

Suomi, S. J. (2005b). How gene-environment interactions shape the development of impulsive aggression in rhesus monkeys. In D. M. Stoff \& E. J. Susman (eds.), Developmental psychobiology of Aggression (252-270). New York: Cambridge University Press.

Susman, E. J., \& Stoff, D. M. (2005). Synthesis and reconsiderations of the psychobiology of aggressive behaviour: A conclusion. In D. M. Stoff \& E. J. Susman (eds.), Developmental psychobiology of Aggression (271-290). New York: Cambridge University Press.

Szyf, M. (2007, March). Epigenetics of human social behaviour. Origin of war and peace: How to teach children to live in a peaceful way. Montreal, Canada: Symposium.

Thapar, A., Holmes, J., Poulton, K., \& Harrington, R. (1999). Genetic basis of attention deficit and hyperactivity. British Journal of Psychiatry, 174, 105111.

Thornberry, T. P. (1997). Developmental theories of crime and delinquency. Advances in criminological theory (vol. 7). New-Brunswick (USA): Transaction publishers.

Tremblay, R. E. (2000). The development of aggressive behavior during chidhood: What have we learned in the past century? International Journal of Behavioral Development, 24 (2), 129-141.

Tremblay, R. E. (2005). Towards an epigenetic approach to experimental criminology: The 2004 Joan McCord Prize Lecture. Journal of Experimental Criminology, 1 (4), 397-415.

Tremblay, R. E. (2006). Tracking the origins of criminal behaviour: back to the future. The Criminologist, 31 (1), 1-7.

Tremblay, R. E., \& Craig, W. M. (1995). Developmental crime prevention. In M. Tonry \& D. P. Farrington (eds.), Building a safer society: Strategic approaches to crime prevention (19, 151-236). Chicago: The University of Chicago Press.

Tremblay, R. E., Vitaro, F., Nagin, D., Pagani, L., \& Séguin, J. R. (2003). The Montreal longitudinal and experimental study: Rediscovering the power of descriptions. In T. Thornberry (ed.), Taking stock of delinquency: An overview of findings from contemporary longitudinal studies (205-254). New York: Plenum.

Tremblay, R. E., Nagin, D. S., Séguin, J. R., Zoccolillo, M., Zelazo, P. D., Boivin, M., Pérusse, D., \& Japel, C. (2004). Physical aggression during early childhood: Trajectories and predictors. Pediatrics, 114 (1), 43-50.

Tremblay, R. E., Hartup, W. W., \& Archer, J. (2005). Developmental origins of aggression. New York: Guilford Press. 
Van Goozen, S. H. M. (2005). Hormones and the developmental origins of aggression. In R. E. Tremblay, W. W. Hartup \& J. Archer, (eds.), Developmental origins of aggression (281-306). New York: Guilford Press.

Viding, E., \& Blakemore, S. J. (2007). Endophenotype approach to developmental psychopathology: Implications for autism research. Behavior Genetics, 37, 51-60.

Vitaro, F., \& Brendgen, M. (2005). Proactive and reactive aggression: A developmental perspective. In R. E. Tremblay, W. W. Hartup \& J. Archer (eds.), Developmental origins of aggression (178-201). New York: Guilford Press.

Voeller, K. K. S. (2006). Attention deficit-hyperactivity disorder. In C. E. Coffey, R. A. Brumback (eds.), Pediatric neuropsychiatry (215-241). Philadelphia: Lippincott Williams \& Wilkins.

Walsh, A. (2003). Intelligence and antisocial behaviour. In A.Walsh, \& L. Ellis (Eds.), Biosocial criminology: Challenging environmentalism's supremacy (105124). New York: Nova Science Publishers.

Walsh, A., \& Ellis, L. (2003). Biosocial criminology: Challenging environmentalism's supremacy. New York: Nova Science Publishers.

Wasserman, D., \& Wachbroit, R. (2001). Genetics and criminal behaviour. Cambridge: Cambridge University Press.

Weaver, I. C. G., Cervoni, N., Champagne, F. A., D’Alessio, A. C., Sharma, S., Seckl, J. R., Dymov, S., Moshe, S., \& Meaney, M. J. (2004). Epigenetic programming by maternal behavior. Nature Neuroscience, 7, 847-854.

Weinhold, B. (2006). Epigenetics. Environmental Health Perspectives, 114, 160167. 